Clemson University

TigerPrints

$9-2019$

\title{
Multiscale Modeling of Superior Cavopulmonary Circulation: Hemi-Fontan and Bidirectional Glenn Are Equivalent
}

\author{
Ethan Kung \\ Chiara Corsini \\ Alison Marsden \\ Irene Vignon-Clemente \\ Giancarlo Pennati
}

See next page for additional authors 


\section{Authors}

Ethan Kung, Chiara Corsini, Alison Marsden, Irene Vignon-Clemente, Giancarlo Pennati, Richard Figliola, and Tain-Yen Hsia 
Full Title: $\quad$ Multiscale Modeling of Superior Cavopulmonary Circulation: Hemi-Fontan And Bidirectional Glenn Are Equivalent

Authors: $\quad$ Ethan Kung ${ }^{1}$; Chiara Corsini²; Alison Marsden 3 ; Irene Vignon-Clementel4; Giancarlo Pennati2; Richard Figliola ${ }^{1,{ }^{*}}$, and Tain-Yen Hsia ${ }^{5,}$; for the Modeling of Congenital Hearts Alliance (MOCHA)+ Investigators

Affiliations:

1. Clemson University, Clemson, SC, USA,

2. Politecnico di Milano, Milan, Italy

3. Stanford University, Stanford, CA

4. National Institute for Research in Computer Science and Automation (INRIA), Paris, France

5. Yale New Haven Children's Hospital, New Haven, CT

$\S$ co-first authors

${ }^{*}$ co-senior authors

MOCHA Investigators: Andrew Taylor, Sachin Khambadkone, Silvia Schievano, and Marc de Leval (Institute of Cardiovascular Sciences, London, UK); Edward Bove, and Adam Dorfman, (University of Michigan, Ann Arbor, MI); G. Hamilton Baker and Anthony Hlavacek (Medical University of South Carolina, Charleston, SC); Francesco Migliavacca, Giancarlo Pennati, and Gabriele Dubini (Politecnico di Milano, Milan, Italy); Alison Marsden, (Stanford University, CA); Irene Vignon-Clementel (National Institute of Research in Informatics and Automation, Paris, France); Richard Figliola and John McGregor (Clemson University, Clemson, SC); Tain-Yen Hsia (Yale University, New Haven, CT)

Total word count: 5924

\section{Funding and Conflicts of Interest:}

30 This work received funding support from Leducq Foundation (France), National Institute of Health Research (UK), Burroughs Wellcome Fund (UK), American Heart Association (US), National Science Foundation (US) and British Heart Foundation (UK). All authors have nothing to disclose regarding possible conflicts of interest.

Corresponding Author:

T-Y Hsia, MD

Pediatric Cardiac Surgery

Yale New Haven Children's Hospital

LCCI 301, 330 Cedar Street

New Haven, CT, USA

Telephone: 1 (203) 737-1298

Email: tain-yen.hsia@yale.edu 
45 Abbreviations

\section{Glossary}

46 artsat-pre preoperative arterial oxygen saturation

47 artsat-post post-operative arterial oxygen saturation

48 bG bi-directional Glenn

49 BSA body surface area

50 CFD computational fluid dynamics

$51 \mathrm{Cl} \quad$ cardiac index

52 CMR cardiac magnetic resonance imaging

53 CS central shunt

54 GA general anaesthetic

$55 \mathrm{Hb} \quad$ hemoglobin

$56 \mathrm{hF}$ hemi-Fontan

57 HLHS hypoplastic left heart syndrome

58 IVC inferior vena cava

59 LPA left pulmonary artery

60 LPN lumped parameter network

61 maxO2cap maximum oxygen capacity

62 mBTS modified Blalock-Taussig shunt

$63 \mathrm{O}_{2}$ del systemic oxygen delivery

$64 \mathrm{O}_{2}$ cons oxygen consumption

65 PA pulmonary arteries

66 PAP pulmonary artery pressure

67 PVR pulmonary vascular resistance

$68 \mathrm{PV}_{\text {sat-pre }} \quad$ pre-operative pulmonary venous oxygen saturation

$69 Q_{p} \quad$ pulmonary blood flow rate

70 Qp-pre pre-operative pulmonary blood flow rate

$71 Q_{s} \quad$ systemic blood flow rate

72 SVC superior vena cava

73 SCPC superior cavopulmonary connection/circulation

74 TPG transpulmonary gradient 
Abstract

77 Objective: Superior cavopulmonary circulation (SCPC) can be achieved by either the 78 Hemi-Fontan $(\mathrm{hF})$ or Bidirectional Glenn (bG) connection. Debate remains as to which 79 results in best hemodynamic results. Adopting patient-specific multiscale computational 80 modeling, we examined both the local dynamics and global physiology to determine if 81 surgical choice can lead to different hemodynamic outcomes.

82 Methods: Six patients (age: 3-6 months) underwent cardiac magnetic resonance imaging 83 and catheterization prior to SCPC surgery. For each patient: 1) a finite 3-dimensional (3D) 84 volume model of the preoperative anatomy were constructed to include detailed definition 85 of the distal branch pulmonary arteries, 2) virtual $\mathrm{hF}$ and bG operations were performed 86 to create two SCPC 3D models, and 3) a specific lumped network representing each 87 patient's entire cardiovascular circulation was developed from clinical data. Using a 88 previously validated multiscale algorithm that couples the 3D models with lumped 89 network, both local flow dynamics, i.e. power loss, and global systemic physiology can be 90 quantified. In two patients whose preoperative imaging demonstrated significant left 91 pulmonary artery (LPA) stenosis, we performed virtual pulmonary arterioplasty to assess 92 its effect.

93 Results: In one patient, the hF model showed higher power loss (107\%) than the bG, 94 while in 3 the power losses were higher in the bG models (18 to $35 \%$ ). In the remaining 95 two patients, the power loss differences were minor. Despite these variations, for all 96 patients, there were no significant differences between the $\mathrm{hF}$ and bG models in hemodynamic or physiologic outcomes, including cardiac output, superior vena cava pressure, right-left pulmonary flow distribution, and systemic oxygen delivery. In the two patients with LPA stenosis, arterioplasty led to better LPA flow (5-8\%) while halving the power loss, but without important improvements in SVC pressure or cardiac output.

101 Conclusion: Despite power loss differences, both hF and bG result in similar SCPC 102 hemodynamics and physiology outcome. This suggests that for SCPC, the pre-existing 103 patient-specific physiology and condition, such as pulmonary vascular resistance, are 
104 more deterministic in the hemodynamic performance than the type of surgical palliation. 105 Multiscale modeling can be a decision assist tool to assess whether an extensive LPA 106 reconstruction is needed at the time of SCPC for LPA stenosis.

$107 \quad$ (Word count $=359)$

108 


\section{Perspective Statement}

110

111 Whether hemi-Fontan or bidirectional Glenn achieves a better superior cavopulmonary 112 circulation remains debatable. Mathematical modelling incorporating both the 3D 113 anatomic detail and systemic physiology can elucidated effects of surgery in a patient 114 specific manner. We applied state of the art computational methods to demonstrate that 115 either palliation led to equivalent hemodynamic outcomes, despite differences in local 116 power loss.

117 Word count: 58 (379 characters)

118

119 Central Message

120

Advanced modeling simulation showed that the hemi-Fontan and bidirectional Glenn

122

123 achieves early equivalent superior cavopulmonary circulation hemodynamics and physiology.

124

125 Word-count: 18 (163 characters)

126 
Introduction

132 Superior cavopulmonary connection (SCPC) is a transitional circulation that allows for 133 volume off-loading of the single ventricle while providing a stable source of pulmonary 134 blood flow that can grow with the patient prior to completing single ventricle palliation with 135 a Fontan procedure. Pioneered by William Glenn at Yale in the 1960's, the classic unidirectional Glenn anastomosis has been replaced by either the bidirectional Glenn (bG) 137 or the hemi-Fontan $(\mathrm{hF})$ procedures. While a $\mathrm{hF}$ facilitates Fontan completion with a 138 lateral tunnel total cavopulmonary connection (TCPC), bG permits creation of an 139 extracardiac TCPC without the need for cardioplegic cardiac arrest. Nonetheless, the choice between the adaptation of either the bG or hF to achieve SCPC remains primarily based on surgeon or institutional preferences. Whereas no direct, randomized comparative study has been performed to demonstrate outcome differences between the two SCPC procedures, the question regarding whether one is superior than the other remains unanswered with two opposing modeling studies highlighting superiority of one versus the other..$^{1,2}$

While methodologically accurate, both of the previous computational modeling investigations focused solely on the local flow dynamics, i.e. the flow and pressures at the $\mathrm{bG}$ or $\mathrm{hF}$ connection. And in so doing, both studies relied on local flow dynamic variables, such as power loss and flow split between the branch pulmonary arteries, to assess performance differences between the bG and hF circulations. However, because the SCPC is just one segment of the global cardiovascular system that is composed of a closed circulatory loop, isolated SCPC modeling with open ended boundary conditions cannot reveal the influence of either the $\mathrm{bG}$ or $\mathrm{hF}$ on the overall systemic physiology such as SVC pressure, cardiac output, and systemic oxygen delivery. Multi-scale modeling combines the strengths of 3D computational fluid dynamics (CFD) with 0-D lumped parameter network to allow comprehensive assessment of hemodynamic effects of the local surgical domain and the global impact on the systemic physiology. Over the last decade, we have used these validated multi-scale models to evaluate a 
161 variety of clinically significant issues and concepts in patients with single ventricle 162 physiology, such as the hybrid procedure for HLHS, branch pulmonary artery stenosis, 163 residual coarctation, systemic-to-pulmonary shunts, exercise physiology, cardiac 164 biomechanics, and alternative initial palliation, and virtual surgery. ${ }^{3-6}$

166 In this study, we conducted an intensive mathematical modeling investigation using 167 clinical data obtained from a cohort of six patients with single ventricle hearts undergoing 168 SCPC procedure to uncover whether the choice between $\mathrm{bG}$ and $\mathrm{hF}$ procedures leads to 169 hemodynamic and physiological differences. In addition to the employment of the multi170 scale modeling scheme with a closed-loop cardiovascular circulation, other novel 171 concepts in this study include: 1) adaptation of patient-specific anatomy (with detailed 172 distal branch pulmonary arteries) and physiologic parameters into the models, 2) 173 performing virtual $\mathrm{bG}$ and $\mathrm{hF}$ procedures based on preoperative magnetic resonance 174 imaging, 3) examining the effects of relieving patient-specific discrete left pulmonary artery 175 (LPA) stenosis at the time of SCPC procedure, and 4) quantifying both hemodynamic and 176 physiologic variables in the context of SCPC. 
178 Patient Selection and Clinical Data

179 Six patients (age: 3-6 month, BSA: 0.26-0.34 $\mathrm{m}^{2}$ ) with single ventricle cardiac defects 180 were enrolled prior to their pre-operative clinical investigations prior to their SCPC 181 procedure. Patients A, E, and F were recruited at the University of Michigan, B and D at 182 the Medical University of South Carolina, and patient C at Great Ormond Street Hospital 183 for Children (GOSH). Institutional review board study approval was obtained for each 184 clinical site and informed consent for the use of clinical data was gained from the 185 participants' legal guardians. The pre-operative clinical details of the six patients are 186 reported in Table 1. Four patients had hypoplastic left heart syndrome (HLHS) and two 187 had a hypoplastic right ventricle. At the stage 1 surgery, patient A received a $3.5 \mathrm{~mm}$ right 188 modified Blalock-Taussig shunt (mBTS), patients B,C,E,F underwent Norwood procedure with $3.5 \mathrm{~mm}$ right $\mathrm{mBTS}$, and patient $\mathrm{D}$ had a $4 \mathrm{~mm} \mathrm{mBTS}$ with left pulmonary arterioplasty.

190 All patient underwent pre-operative cardiac magnetic resonance imaging (CMR), cardiac 191 catheterization and echocardiography prior to surgery. Depending on institutional 192 preferences, CMR was either performed immediately prior to surgery under the same 193 general anesthesia (GA), on the day of cardiac catheterization under the same GA with 194 transfer between imaging suites, or in a hybrid CMR catheterization imaging suite. CMR 195 was performed on 1.5T scanners (Philips Intera Achieva, Best, Netherlands; Siemens 196 Avanto, Siemens Medical Solutions, Erlangen, Germany). Contrast enhanced CMR 197 angiography was performed to obtain three-dimensional (3D) anatomical imaging with a 198 routine clinical sequence using $0.2 \mathrm{mmol} / \mathrm{kg}$ of intravenous gadoteridol (Prohance; Bracco 199 Diagnostics, Princeton, NJ). Free-breathing, electrocardiogram-gated velocity-encoded 200 phase contrast imaging sequences were used to acquire flow measurements in the 201 ascending and descending aorta, pulmonary arteries and veins, and inferior (IVC) and 202 superior vena cavae (SVC). 
204 Cardiac catheterization followed a routine clinical protocol under GA or sedation in a bi205 plane fluoroscopy suite (Siemens Medical Solutions USA, Inc. Pennsylvania). A fluid206 filled catheter system was used to acquire pressure traces and hemodynamic 207 measurements in the ascending and descending aorta, systemic atrium, and single 208 ventricle. Pulmonary artery pressure (PAP) was either a direct measurement or an 209 estimate from pulmonary venous wedge pressure. In patients $C$, E, and F, PAP was 210 acquired on the left side, with no clinical evidence suggestive of a stenosis or cause for 211 discrepancy between the two pulmonary arteries (PA). In patients A, B, and D, PAPs were 212 acquired on the left and right sides. Patient $B$ also had left pulmonary artery stenosis 213 (PAS), and patient $D$ had left PAS with a $3 \mathrm{mmHg}$ pressure difference between the two 214 pulmonary arteries. Only in patient $D$ did the clinical team felt a left pulmonary 215 arterioplasty was indicated. Pre-operative echocardiography was performed under GA or 216 sedation. Pulsed wave Doppler traces were acquired in the aorta, SVC, IVC and branch 217 PAs.

218 All clinical data processing occurred at one core laboratory (GOSH). A representation of 219 the patients' pre-operative physiology was constructed from CMR flows and invasive 220 mean pressure measurements. The resulting parameters presented in Table 2 were used 221 to tune the multiscale models as described below. Flow measurements were calculated 222 using an in-house plug-in for OsiriX open-source software (OsiriX Foundation, Geneva, 223 Switzerland). These are reported indexed to BSA to aid comparison between patients.

224 Three-dimensional (3D) models and virtual surgery

$2253 \mathrm{D}$ models of each patients' stage 1 anatomy were reconstructed from the CMR 226 angiographic sequences using commercially available software (Mimics, Materialise NV, 227 Leuven, Belgium). In Figure 1, we illustrate the reconstruction process using Patient $B$ as 228 an example, while Figure 2 depicts the stage 1 and stage 2 reconstructions for the six 229 patients studied. Referring to Figure 1, a region of interest was selected within the relevant 230 area of surgical anatomy. A 3D geometrical model was constructed through a process of 231 region-growing and segmentation. ${ }^{7,8}$ The pre-operative 3D model for each patient 232 included the mBTS and PAs extended to the furthest branch level visible for reconstruction 
233 (Figure 1, stage 1). The location of the SVC and atrium was noted for construction of the 234 stage 2 virtual surgery. To this end, the stage 1 geometry was manipulated virtually, 235 removing the $\mathrm{mBTS}$ and inserting the reconstructed SVC in its stead, merging the volumes 236 with a Boolean operation (Figure 1, stage 2). In the case of $\mathrm{hF}$, a portion of the atrium was 237 reconstructed from the original CMR dataset and similarly merged in the 3D domain, again 238 after removal of the mBTS. In both cases, where appropriate, a pulmonary arterioplasty 239 model was generated by virtually enlarging the caliber of the narrowed PA. Prior to use 240 for stage 2 simulations, the realistic nature of all virtual surgery models shown in Figure 2 241 was verified by the surgeons (EB, TYH) involved in the study.

242

243 Multiscale Simulation and Analysis

244 Multi-scale models were developed and tuned for each patient based on the patient245 specific anatomical and clinical data (Table 2). According to our previous work, ${ }^{3,4}$ we 246 constructed a OD LPN to model the circulatory system outside of the surgical region, which 247 was coupled directly to the inflow and outflow passages of the 3D model of the surgical 248 site. Briefly, the closed-loop LPN includes sections that describe the heart, upper and 249 lower body vasculatures, pulmonary vasculature, and vascular beds in several abdominal 250 organs. The contraction and filling of each heart chamber is described via a passive and 251 active pressure-volume curve and an activation function. ${ }^{3,4}$ This allows the simulation to 252 capture effects of preload on cardiac output due to the Frank-Starling mechanism. The 253 influence of respiration was neglected for this study.

254

255 Each patient was modeled at the age and body surface area (BSA) at the time of their 256 CMR scan since both 3D and flow information is acquired at this time-point. As described 257 in our prior work, most elements of the LPN were tuned initially using reference values 258 (that were scaled by allometric equations based on each patient's particular BSA and then 259 further adjusted for each patient based on available clinical data. ${ }^{9-12}$ The LPN parameters 260 in the pulmonary vasculature were automatically estimated based on multiscale 261 preoperative simulations to match the relevant clinical measurements. ${ }^{13}$ We divided the 262 pulmonary vasculature into several parts to be represented as lumped components. 
263 These parts include the large arteries, smaller arteries, capillaries, and veins; Next, 264 empirical laws determined the distribution of the equivalent resistance and capacitance 265 over the arterial or venous sides. ${ }^{14-16}$ Windkessel models were generated from the 266 Womersley-based impedance of each pulmonary branch, ${ }^{17,18}$ therefore the proximal to 267 distal pulmonary artery resistance ratio is different for each branch. Combining all of these 268 relations provided a unique set of LPN parameters for each pulmonary branch based on 269 its total resistance.

270 Multi-scale simulations of the post-operative scenarios were conducted according to 271 previously validated techniques. 7, 12, 19, 20 Briefly, this involves discretizing the 3D virtual 272 surgery geometries into isotropic finite-element meshes with maximum edge size of 0.03 $273 \mathrm{~cm}$ (MESHSIM, Simmetrix Inc., New York) and coupling the 3D Navier-Stokes equations 274 to the OD LPN using Neumann boundary conditions, implicit coupling, and outflow 275 stabilization. ${ }^{21}$ Flow and pressure in the 3D and LPN domain were solved using a custom 276 incompressible finite element Navier-Stokes solver (Simvascular, www.simtk.org), and a $2774^{\text {th }}$ order Runge-Kutta algorithm, respectively. Simulation time step size was $1 \mathrm{~ms}$ and 1 $278 \mu$ s for the 3D and LPN domain, respectively. Flow and pressure coupling between 279 domains occurs at every 3D time step. Each simulation included 12 cardiac cycles where 280 the last cycle data, by which periodicity had been achieved, was used in the final results 281 analysis.

282 Power loss was calculated from the simulation results according to our previous 283 publication. ${ }^{4}$ To summarize, the surgical junction power loss was obtained from the 3D 284 data by integrating the sum of inlet and outlet face energy fluxes, which accounts for both 285 the potential and kinetic energy. The power loss across a vascular bed was obtained from 286 the OD data by multiplying the pressure drop and the total flow across the vascular bed.

287 Post-operative predictions of systemic oxygen delivery, and arterial and venous 288 saturations were calculated using a combination of pre-operative clinical measurements 289 and post-operative predictions of flow. We assumed: 1) the pre-operative estimates of 290 maximum oxygen capacity and oxygen consumption remained the same immediately 
291 following surgery; 2) pulmonary venous saturations remain the same immediately post292 operatively; and 3) the relative upper and lower body oxygen consumption after surgery 293 is directly proportional to flow. The maximum oxygen carrying capacity of blood $\operatorname{maxO}_{2}$ cap $294\left(\mathrm{mlO}_{2} / 100 \mathrm{ml}\right)$ was estimated as

295

$$
\max \mathrm{O}_{2} \mathrm{cap}=H b_{\text {pre }} \leftrightarrow .34
$$

296 where $H b_{\text {pre }}$ is pre-operative hemoglobin in $\mathrm{g} / \mathrm{dL}$ and 1.34 represents Hüfner's constant (a 297 directly measured estimate of the maximum oxygen carrying capacity of blood equal to $2981.34 \mathrm{ml} \mathrm{O} 2 / \mathrm{g}$ of hemoglobin). The oxygen consumption $\mathrm{O}_{2}$ cons $\left(\mathrm{mlO}_{2} / \mathrm{min} / \mathrm{m}^{2}\right)$ was 299 estimated as

300

$$
O_{2} \text { cons }=Q_{p-p r e} \leftrightarrow \frac{P V_{\text {sat-pre }}-a r t_{\text {sat }- \text { pre }}}{100} \leftrightarrow \max O_{2} \text { cap } \leftrightarrow 0 / B S A
$$

301 where $Q_{p-p r e}$ is the pre-operative measured pulmonary flow (L/min), $P V_{\text {sat-pre }}$ is the 302 measured pre-operative pulmonary venous oxygen saturations (\%), artsat-pre is the 303 measured arterial oxygen saturations (\%), and BSA is body surface area $\left(\mathrm{m}^{2}\right)$. The post304 operative estimated systemic oxygen delivery $\mathrm{O}_{2} \mathrm{del}\left(\mathrm{mlO}_{2} / \mathrm{min} / \mathrm{m}_{2}\right)$ was calculated as

305

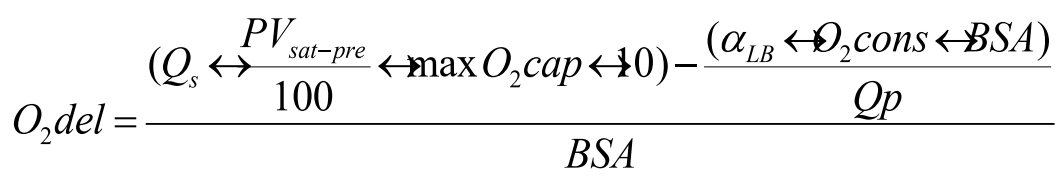

306 where $Q_{s}$ is systemic flow ( $\left./ \mathrm{min}\right)$ calculated from the post-operative simulation, $\alpha_{L B}$ is the 307 proportion of $Q_{s}$ to the lower body based on post-operative simulation results, and $Q_{p}$ is 308 the post-operative pulmonary flow $(\mathrm{L} / \mathrm{min})$ from simulation results. The post-operative 309 arterial oxygen saturation artsat-post $(\%)$ was estimated as

$$
\operatorname{art}_{\text {sat-post }}=\frac{\mathrm{O}_{2} \mathrm{del}}{Q_{S}} \stackrel{\mathrm{BSA}}{\max \mathrm{O}_{2} \operatorname{cap} \leftrightarrow 0}
$$




\section{Results}

313 There were significant differences in local flow patterns and pressure distributions 314 between the two surgical options. Using the results for patient $B$ as an example (Figure 3153 ), the bG geometry typically reveals a flow jet of blood from the SVC that impinges on 316 the bottom of the PA wall at the anastomosis where it divides to the branches (Figure 3, $317 \mathrm{~A}$ and $\mathrm{C}$ ). However, in the $\mathrm{hF}$ geometry there is slight vortex of blood as the incoming SVC 318 flow glides along the atrial wall (Figure 3, B and D). In the cases where an LPA stenosis 319 is present (Figure 3, A and B), there is also a flow jet following and a nominal $1 \mathrm{mmHg}$ pressure loss across the stenosis. The pressure and flow patterns predicted here were consistent with those of a previous study on patients at the same stage but right before the Fontan surgery. ${ }^{18}$

323 The local power loss in the surgical SCPC junction (Table 3, 3D power loss) varied 324 considerably between bG and hF models. In four patients, hF showed a notably higher power loss (18 to $107 \%$ ) than bG , which was consistent with the differences in flow fields and pressures between these differing surgical options. In two patients, power losses were essentially equivalent ( $<7 \%$ difference) between options. In comparing the 3D power loss with the total power loss across the entire pulmonary vascular bed (Table 3), the amount of power loss occurring within the SCPC junction is only 1 to $16 \%$ of that across the entire pulmonary circulation. The magnitudes of these power losses are compared 331 directly in Figure 4. Much larger differences in the total pulmonary power loss exist between patients with different pulmonary vascular resistances (PVR) than between the different surgical options of the same patient.

334 As a consequence, the post-operative SCPC simulation results revealed similar 335 physiologic outcomes between the various surgical options for each patient (Table 3 ). The bG and hF surgical models had small differences in transpulmonary gradient (TPG) and 337 SVC pressure (Psvc) (up to $5 \%$ and $2 \%$, respectively), and negligible differences in cardiac 338 index $(\mathrm{Cl})(<1 \%)$ with nearly identical pulmonary to systemic flow splits. Oxygen delivery

339 (Table 3) closely followed cardiac index for each patient, and both oxygen delivery and 
340 oxygen saturations were insensitive to the surgical option for a specific patient.

341 The power losses in models with left PAS were found to be more than two times higher $342(217-248 \%)$ than that in models where the left PAS was relieved by virtual arterioplasty. 343 While the relief of PAS led to slightly better LPA flow (5-8\%) in both patients, there was 344 no important improvement in SVC pressure or cardiac output compared to models where 345 the left PAS was left intact. 
Discussion

348 In 1996, Marc de Leval and his collaborators in Milan, Italy reported the first instance where computational fluid dynamics (CFD), a relatively new engineering field, was applied to the evaluation of a reconstructive cardiac procedure. ${ }^{24}$ While the mathematics were sophisticated and investigation revealed interesting flow dynamic insights in the TCPC, the problem facing the investigators was how to translate the mathematical information to the surgical community. They needed a parameter or variable that a congenital cardiac surgeon can appreciate its value and correlate with clinical significance. Since the ability of CFD at that time only allowed for modeling an isolated surgical domain, i.e. the cavopulmonary connections, their models required compulsory open-ended inlets and outlets with rigid, prescribed boundary conditions. And in such an open-loop circulatory model, only the local pressure and flow conditions can be quantified, leaving any interaction with the rest of the global, systemic circulation unanswered. Therefore, as a way to quantify the 'performance' of a cavopulmonary connection, the concept of power loss was introduced to describe the extraction of fluid dynamic power, or energy, as blood traversed from the inlets (vena cava) to the outlets (branch pulmonary arteries). Less power loss in a cavopulmonary circulation, the better. And power loss became the goal post for which future modeling investigations of the cavopulmonary circulation would be based on. 1,2,18,22

In this study, where a closed loop circulatory model allowed for interaction between the SCPC and the rest of the cardiovascular system, our patient-specific multi-scale simulations showed that differences in power loss between a hF and bG SCPC, even when greater than two-fold, resulted in negligible effect on clinically relevant parameters such as cardiac index, SVC pressure, and systemic oxygen delivery. Therefore, either hF or bG, as the procedure of choice for SCPC, would achieve similar hemodynamic and physiologic results. This is unlikely to be a controversial finding, as most surgeons would agree that outcomes after either procedure have been viewed to be similar. Nonetheless,

374 by employing the state of art multi-scale modeling with patient-specific anatomy and physiology information, this study should settle the hF versus bG debate while highlighting 376 the importance of evaluating the hemodynamic performance of a cardiac surgical 
377 procedure, not in isolation, but in context of the global systemic circulation.

378 So, why is power loss differences between $\mathrm{hF}$ and bG not important? This can be 379 explained by examining the SCPC junction power loss in the context of the systemic 380 circuit. An advantage of multi-scale computational modeling is this inclusion of the patient381 specific systems-level physiology on the predicted hemodynamic outcomes. Due to the 382 fact that only a small fraction of the power loss in the pulmonary circulation actually occurs 383 over the SCPC junction, change in the junction power loss by several folds can still only 384 have limited effects on the overall circulation. As noted, Figure 4 illustrates the 385 contribution of the SCPC junction power loss to the total pulmonary power loss in each patient. It is clear that much of the power loss via the pulmonary circulation occurs outside 387 of the surgical junction, meaning that the patient PVR has a much larger impact on the 388 overall physiology than the hemodynamic differences between $\mathrm{hF}$ and bG. We observed 389 the same relative significance between the SCPC and total pulmonary power loss in all 6 390 patients. There is a caveat, however: this does not mean power loss does not matter at 391 all. In situations or conditions where there is important lesion that impacts on blood flow, 392 such as severe LPA stenosis or SCPC anastomotic obstruction, the power loss through a 393 cavopulmonary connection can become high enough to be on similar order as PVR and 394 there will be adverse hemodynamic consequences. Also, under higher metabolic states, 395 such as exercise, as flow increases and PVR drops, the ratio between the SCPC and the 396 total pulmonary power loss is expected to rise. Further studies will be needed to 397 determine whether this would result in more noticeable differences in physiology between 398 different surgical geometries.

399 The multi-scale simulations also revealed that in two patients with discrete left PA 400 stenosis, virtual augmentation with arterioplasty did not lead to important benefits in the 401 overall performance and hemodynamics of the SCPC. This suggests that, in these two 402 patients, a more extensive and potentially risky operation (i.e. division of the Damus-Kay403 Stansel anastomosis to get to the left PA) to relieve LPA stenosis would not have led to 404 additional hemodynamic and physiologic benefits. Again, this discovery cannot be applied 405 to all instances of left PA stenosis, as surely relief of severe left PA stenosis is important 
406 at the time of a cavopulmonary connection procedure. Nonetheless, these simulation 407 results suggests that not all left PA stenosis requires extensive arterioplasty, and a 408 combination of virtual surgery with multi-scale modeling can provide valuable support and 409 guidance to a surgeon's decision on whether a patient-specific left PA stenosis can be left 410 untouched at the time of SCPC.

411 Closed loop modeling of stage 1 physiology represents a challenge due to the complexity 412 of the physiology and time-varying nature of the hemodynamic measurements. Clinical 413 data is acquired at different time points and used to build a representation of the patient's 414 pre-operative physiology. The aim of this study was to compare the effect of different 415 surgical anatomies, without additional adaptation from the global physiology. 416 Consequently, responses such as post-operative stress response, effects of medication, 417 chronic adaption to new ventricular loading conditions, post-operative complications, and 418 the effects of growth on the clinical data are not modeled. In light of this, validation of the 419 predicted results against existing clinical data remains limited. The simulations represent 420 a prediction of the immediate post-operative physiology based on the physiological 421 impacts of loading changes induced by the surgical procedures only. A direct comparison 422 between the surgical options is essential for gaining a mechanistic understanding of the 423 hemodynamics in the relevant clinical scenarios. One step towards assessing the 424 robustness of the predicted results would be to incorporate approaches that also contain 425 sensitivity analysis ${ }^{18}$ or uncertainty quantification ${ }^{23}$, including both the clinical data and 426 physiological model parameters. This might be especially important when preoperative 427 clinical data are not coherent. ${ }^{13}$ Currently, such methods are computationally expensive 428 and in need of further development. ${ }^{23}$ It is also important to point out that while variation 429 in the bi-directional Glenn procedure is limited, the construction of a hemi-Fontan can be 430 quite variable from one institution and one surgeon to another. As only one institution 431 (Michigan) in our collaboration routinely applied the hemi-Fontan, we have adopted virtual 432 hemi-Fontan models without additional patch enlargement of the left pulmonary artery was 433 described by William Norwood. Therefore, it is possible that an left PA stenosis will 434 routinely be addressed by this manner of hemi-Fontan construction. Finally, any virtual 435 surgery and computational modeling investigation, even using patient specific information, 
436 cannot account for all the biological and clinical processes that impacts on ultimate 437 outcome. Therefore, the findings from this study should be applied in the context of clinical 438 decision-making support.

439

440

\section{Conclusion}

441 In this first case series of patient-specific multiscale modeling of superior cavopulmonary 442 connection palliation for single ventricle hearts, virtual hemi-Fontan and bidirectional 443 Glenn procedures were simulated based on each patient's preoperative anatomy and 444 physiologic conditions derived from clinically indicated investigations. Despite what 445 appeared to be significant local power loss differences, both the hemi-Fontan and 446 bidirectional Glenn procedures resulted in similar early postoperative superior 447 cavopulmonary hemodynamics and physiology. Moreover, simulations suggest that multi448 scale modeling may be helpful to support patient-specific decision on whether an 449 aggressive left pulmonary artery reconstruction at the time of SCPC procedure could be 450 beneficial. 
453

454 The authors gratefully acknowledge support from Foundation Leducq.

455

456 The authors have nothing to disclose with regard to commercial support. 
459 1. Bove EL, de Leval MR, Migliavacca F, Guadagni G, Dubini G, Computational fluid dynamics in the evaluation of hemodynamic performance of cavopulmonary connections after the Norwood procedure for hypoplastic left heart syndrome. $J$ Thorac Cardiovasc Surg 126, 1040-1047 (2003).

2. Pekkan $\mathrm{K}$ et al., Hemodynamic Performance of Stage-2 Univentricular Reconstruction: Glenn vs. Hemi-Fontan Templates. Ann Biomed Eng 37, 50-63 (2009).

3. Corsini $\mathrm{C}$ et al., An integrated approach to patient-specific predictive modeling for single ventricle heart palliation. Comput Methods Biomech Biomed Engin, (2013).

468

469

470

4. Kung $E$ et al., Predictive modeling of the virtual Hemi-Fontan operation for second stage single ventricle palliation: Two patient-specific cases. J Biomechanics 46, 423-429 (2013).

5. Schiavazzi DE et al., Hemodynamic effects of left pulmonary artery stenosis after superior cavopulmonary connection: A patient-specific multiscale modeling study. J Thorac Cardiovasc Surg 149, 689-696.e683 (2015).

6. Vignon-Clementel IE, Marsden AL, Feinstein JA. A primer on computational simulation in congenital heart disease for the clinician. Prog. Pediatr. Cardiol. 30(1), 3-13, 2010.

7. Corsini $C$ et al., An integrated approach to patient-specific predictive modeling for single ventricle heart palliation. Comput Methods Biomech Biomed Engin, (2013).

8. Schievano $S$ et al., Percutaneous pulmonary valve implantation based on rapid prototyping of right ventricular outflow tract and pulmonary trunk from MR data. Radiology 242, 490-497 (2007).

9. Snyder MF, Rideout VC, 1969. Computer simulation studies of the venous circulation. IEEE Transactions on Biomedical Engineering 16, 325-334.

10. Noordergraaf A, Verdouw D, Boom HB, 1963. The use of an analog computer in a circulation model. Progress in Cardiovascular Diseases 5, 419-439.

11. Pennati G, Fumero R, 2000. Scaling approach to study the changes through the gestation of human fetal cardiac and circulatory behaviors. Annals of Biomedical Engineering 28, 442-452. 
504

505

506

507

508

509

510

511

512

513

514

515

516

517

518

519

520

521

522

523

524

525

12. Kung E et al., Predictive modeling of the virtual Hemi-Fontan operation for second stage single ventricle palliation: Two patient-specific cases. J Biomech 46, 423-429 (2013).

13. Arbia, G., Corsini, C., Baker, C., Pennati, G., Hsia, T.-Y., Vignon-Clementel, I., 2015. Pulmonary hemodynamics simulations before stage 2 single ventricle surgery: Patient-specfic parameter identification and clinical data assessment. Cardiovascular Engineering and Technology, 1-13.

14. Brody JS, Stemmler EJ, DuBois AB. Longitudinal distribution of vascular resistance in the pulmonary arteries, capillaries, and veins. J. Clin. Invest. 47, 783-799 (1968). DOI 10.1172/JCI105773

15. O'Leary CE, Fiori R, Hakim TS. Perioperative distribution of pulmonary vascular resistance in patients undergoing coronary artery surgery. Anesth. Analg. 82, 958-963 (1996)

16. Presson RG, Audi SH, Hanger CC, Zenk GM, Sidner RA, Linehan JH, Wagner WW, Dawson CA. Anatomic distribution of pulmonary vascular compliance. J. Appl. Physiol. 84, 303-310 (1998)

17. Spilker RL, Feinstein JA, Parker DW, Reddy VM, Taylor CA, 2007. Morphometrybased impedance boundary conditions for patient-specific modeling of blood flow in pulmonary arteries. Annals of Biomedical Engineering 35, 546-559.

18. Troianovski G., Taylor CA, Feinstein JA, Vignon-Clementel IE. 2011. Threedimensional simulations in Glenn patients: clinically based boundary conditions, hemodynamic results and sensitivity to input data. Journal of Biomechanical Engineering 133, 111006.

19. Baker CE et al., Effects of pulmonary artery banding and retrograde aortic arch obstruction on the hybrid palliation of hypoplastic left heart syndrome. J Thorac Cardiovasc Surg, (2013).

20. Hsia TY et al., Use of mathematical modeling to compare and predict hemodynamic effects between hybrid and surgical Norwood palliations for hypoplastic left heart syndrome. Circulation 124, S204-210 (2011).

21. Esmaily Moghadam M., Bazilevs Y, Hsia TY, Vignon-Clementel IE, Marsden AL, A comparison of outlet boundary treatments for prevention of backflow divergence with relevance to blood flow simulations. Computational Mechanics, 1-15 (2011).

22. Dasi, L. P., KrishnankuttyRema, R., Kitajima, et al.. Fontan hemodynamics: importance of pulmonary artery diameter. J Thoracic Cardiovasc Surg, 137(3), 560- 
564. (2009)

527

528

529

530

531

532

533

534

535
23. Schiavazzi DE, Arbia G, Baker C, Hlavacek AM, Hsia TY, Marsden AL, VignonClementel IE, et al. Uncertainty quantification in virtual surgery hemodynamics predictions for single ventricle palliation. International Journal for Numerical Methods in Biomedical Engineering, 2015.

24. de Leval MR, Dubini G, Migliavacca F, Jalali H, Camporini G, Redington A, Pietrabissa R. Use of computational fluid dynamics in the design of surgical procedures: application to the study of competitive flows in cavo-pulmonary connections. J Thorac Cardiovasc Surg. 1996 Mar;111(3):502-13. 
536

537

538 List of Tables

539 Table 1: Pre-operative demographics of the six patients used for the study.

540 Table 2: Clinical parameters used for pre-operative multiscale modeling.

541 Table 3: Post-operative predictions. 
Table 1: Pre-operative demographics of the six patients used for the study

\begin{tabular}{|c|c|c|c|c|c|c|}
\hline Patient & $\bar{A}$ & B & $\bar{C}$ & $\bar{D}$ & $\bar{E}$ & $\bar{F}$ \\
\hline $\begin{array}{c}\text { Age }^{*} \\
\text { (months) }\end{array}$ & 6 & 3 & 4 & 3 & 4 & 5 \\
\hline BSA $\left(m^{2}\right)$ & 0.34 & 0.30 & 0.27 & 0.26 & 0.28 & 0.34 \\
\hline Diagnosis & PA/IVS & MS, AS & MS, AS & $\begin{array}{l}\text { TA, PA } \\
\text { left PAS }\end{array}$ & $\mathrm{MA}, \mathrm{AA}$ & MA, AA \\
\hline $\begin{array}{l}\text { Stage } 1 \\
\text { surgery }\end{array}$ & $\begin{array}{l}3.5 \mathrm{~mm} \\
\mathrm{mBTS}\end{array}$ & $\begin{array}{l}\text { Norwood } \\
3.5 \mathrm{~mm} \\
\text { mBTS }\end{array}$ & $\begin{array}{l}\text { Norwood } \\
3.5 \mathrm{~mm} \\
\text { mBTS }\end{array}$ & $\begin{array}{l}4 \mathrm{~mm} \text { mBTS } \\
\text { with } \\
\text { LPAplasty }\end{array}$ & $\begin{array}{c}\text { Norwood } \\
3.5 \mathrm{~mm} \\
\text { mBTS }\end{array}$ & $\begin{array}{c}\text { Norwood } \\
3.5 \mathrm{~mm} \\
\text { mBTS }\end{array}$ \\
\hline
\end{tabular}

*Age used for model construction

BSA: body surface area; PA/IVS: pulmonary atresia/intact ventricular septum; mBTS: modified Blalock-Taussig shunt; MS: mitral stenosis; AS: aortic stenosis; TA: tricuspid atresia, PAS: pulmonary artery stenosis; MA: mitral atresia; AA: aortic atresia 
Table 2: Clinical parameters used for pre-operative multiscale modeling

\begin{tabular}{|c|c|c|c|c|c|c|}
\hline Patient & $\bar{A}$ & $\bar{B}$ & $\overline{\mathbf{C}}$ & D & $\bar{E}$ & $\bar{F}$ \\
\hline $\begin{array}{c}\mathrm{Cl} \\
\left(\mathrm{L} / \mathrm{min} / \mathrm{m}^{2}\right)\end{array}$ & 4.31 & 4.08 & 6.87 & 6.23 & 5.79 & 5.47 \\
\hline $\begin{array}{c}\text { Qp } \\
\left(L / m i n / m^{2}\right)\end{array}$ & 1.32 & 1.94 & 3.69 & 2.77 & 2.57 & 3.53 \\
\hline $\begin{array}{c}\text { Qrpa : Qp } \\
(\%)\end{array}$ & 64 & 46 & 51 & 67 & 46 & 55 \\
\hline Qp : Qs & 0.44 & 0.91 & 1.16 & 0.80 & 0.80 & 1.81 \\
\hline $\begin{array}{c}\mathrm{mP}_{\text {atr }} \\
(\mathrm{mmHg})\end{array}$ & 5 & 6 & 6 & 7 & 5 & 4 \\
\hline $\begin{array}{c}\text { mPAP } \\
(\mathrm{mmHg})\end{array}$ & $\begin{array}{l}\text { 13(R), } \\
12(\mathrm{~L})\end{array}$ & $12(\mathrm{R} / \mathrm{L})$ & 11(L) & $\begin{array}{c}17(\mathrm{R}), \\
14(\mathrm{~L})\end{array}$ & $13(\mathrm{~L})$ & $13.5(\mathrm{~L})$ \\
\hline $\begin{array}{c}\text { TPG } \\
(\mathrm{mmHg})\end{array}$ & 8 & 6 & 5 & 10 & 8 & 9.5 \\
\hline $\begin{array}{c}\mathrm{mP}_{\text {Ao }} \\
(\mathrm{mmHg})\end{array}$ & $43^{\#}$ & 52 & 51 & 53 & 53 & 72 \\
\hline $\begin{array}{c}\text { PVR } \\
\left(\mathrm{WU}^{*} \mathrm{~m}^{2}\right)\end{array}$ & 6.0 & 3.1 & 1.4 & 3.6 & 2.8 & 2.7 \\
\hline $\begin{array}{c}\text { SVR } \\
\left(W U^{*} m^{2}\right)\end{array}$ & 12.7 & 21.5 & 14.2 & 13.3 & 14.8 & 35.0 \\
\hline
\end{tabular}

\#estimated from left upper limb sphygmomanometer

$\mathrm{Cl}$ : cardiac index; Qp and Qs: pulmonary and systemic flow; Qrpa: right pulmonary artery flow; $\mathrm{mPatr}$ : mean systemic atrial pressure; mPAP: mean pulmonary artery pressure; R/L: right/left; TPG: transpulmonary gradient; $\mathrm{mP}_{\mathrm{A}_{0} \text { : }}$ mean invasive ascending aortic pressure; PVR and SVR: pulmonary and systemic vascular resistance. 


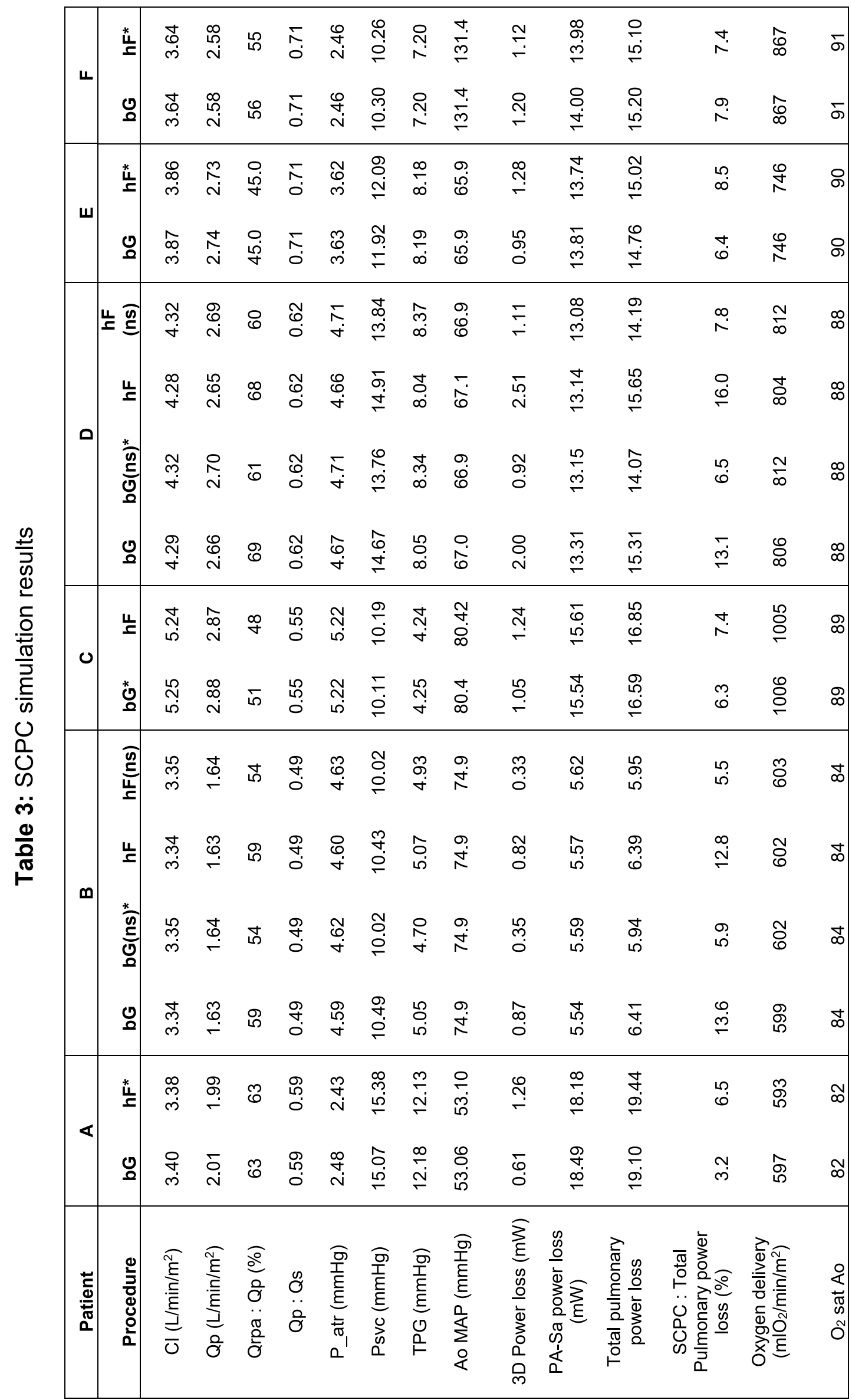

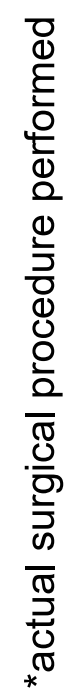




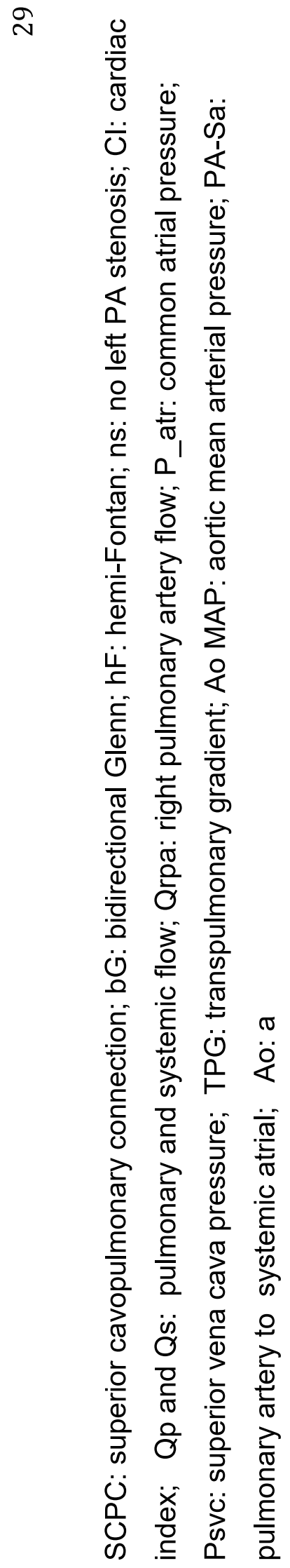




\section{Figure Legends}

Figure 1. An example of the virtual $b G$ and $h F$ superior cavopulmonary surgeries using patient B. Top panel demonstrates the preoperative (Stage 1) anatomy obtained from MRI. Middle panel demonstrates virtual $b G$ and $\mathrm{hF}$ surgeries. Bottom panel demonstrates virtual $\mathrm{bG}$ and $\mathrm{hG}$ procedures with concomitant left pulmonary arterioplasty for relieve of left pulmonary arterial stenosis. SVC: superior vena cava; BTS: Blalock-Taussig shunt; atr: atrium; bG: di-directional Glenn; hF: hemiFontan; PA: pulmonary artery

Figure 2. The preoperative anatomical reconstruction (stage 1) and virtual surgery (stage 2) for all six patients studied. Patients B and D had left pulmonary arterial stenosis preoperatively, and virtual SCPC surgeries were performed with and without concomitant left pulmonary arterioplasty. bG: di-directional Glenn; hF: hemi-Fontan; PA: pulmonary artery

Figure 3: Mid-systolic pressure and velocity maps for patient B. Panel A: bidirectional Glenn with left pulmonary artery stenosis; panel B: hemiFontan with left pulmonary artery stenosis; panel C: bi-directional Glenn with pulmonary arterioplasty; panel $\mathrm{D}$ : hemi-Fontan with pulmonary arterioplasty.

Figure 4. A comparison of the power loss in the SCPC surgical junction and the total power loss through the pulmonary circulation. Power loss through the SCPC surgical junction, whether bG or hF, represents a very small part of the overall power loss in the superior cavopulmonary circulation. bG: di-directional Glenn; hF: hemi-Fontan; PA: pulmonary artery; ns: no pulmonary arterial stenosis; mW: milli-Watts. 


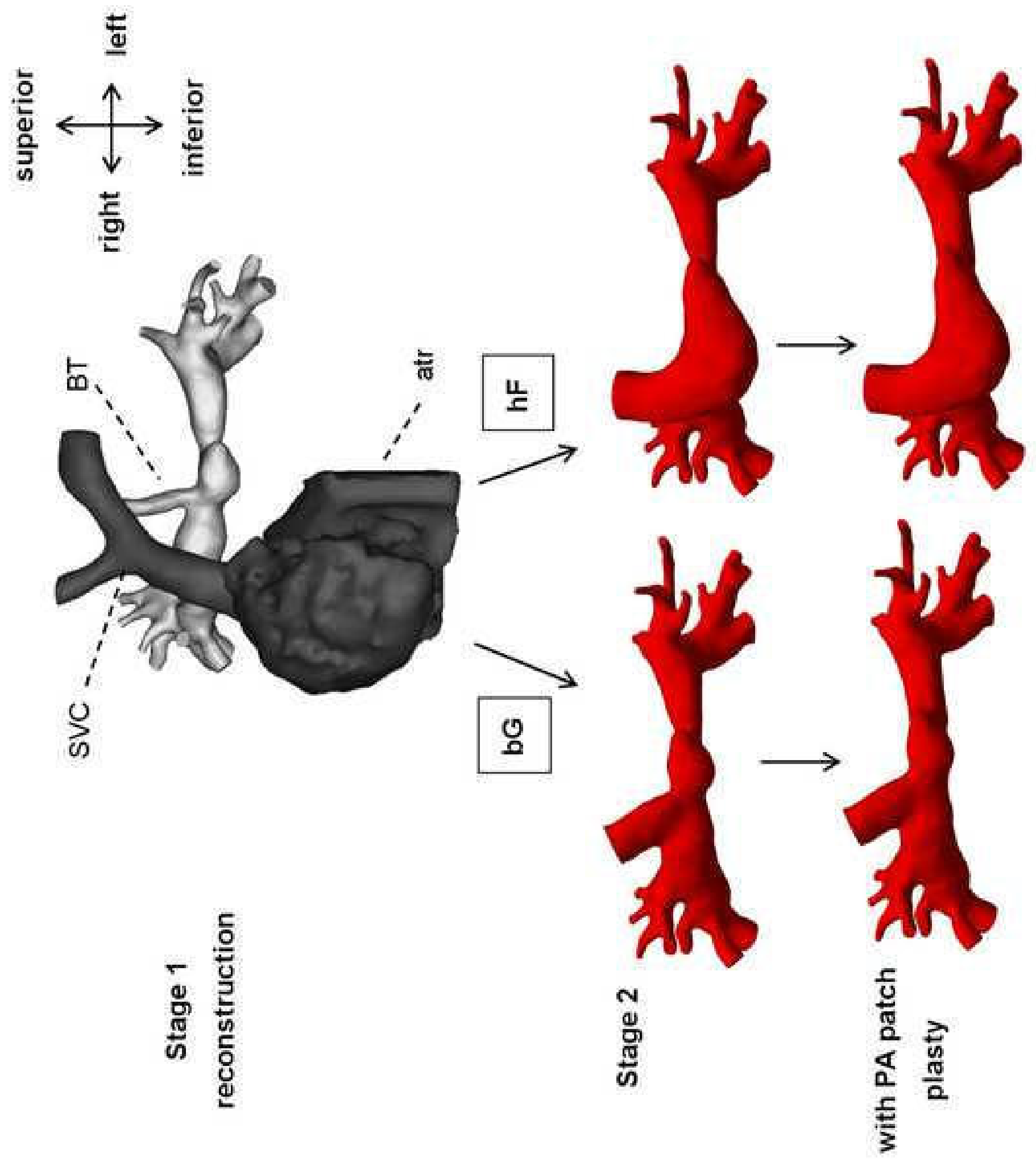

$\frac{0}{3}$
$\frac{0}{0}$
$\overline{0}$
$\overline{0}$ 


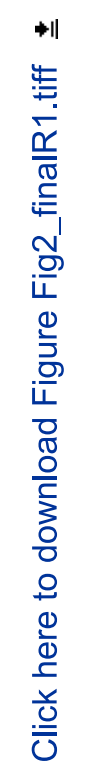
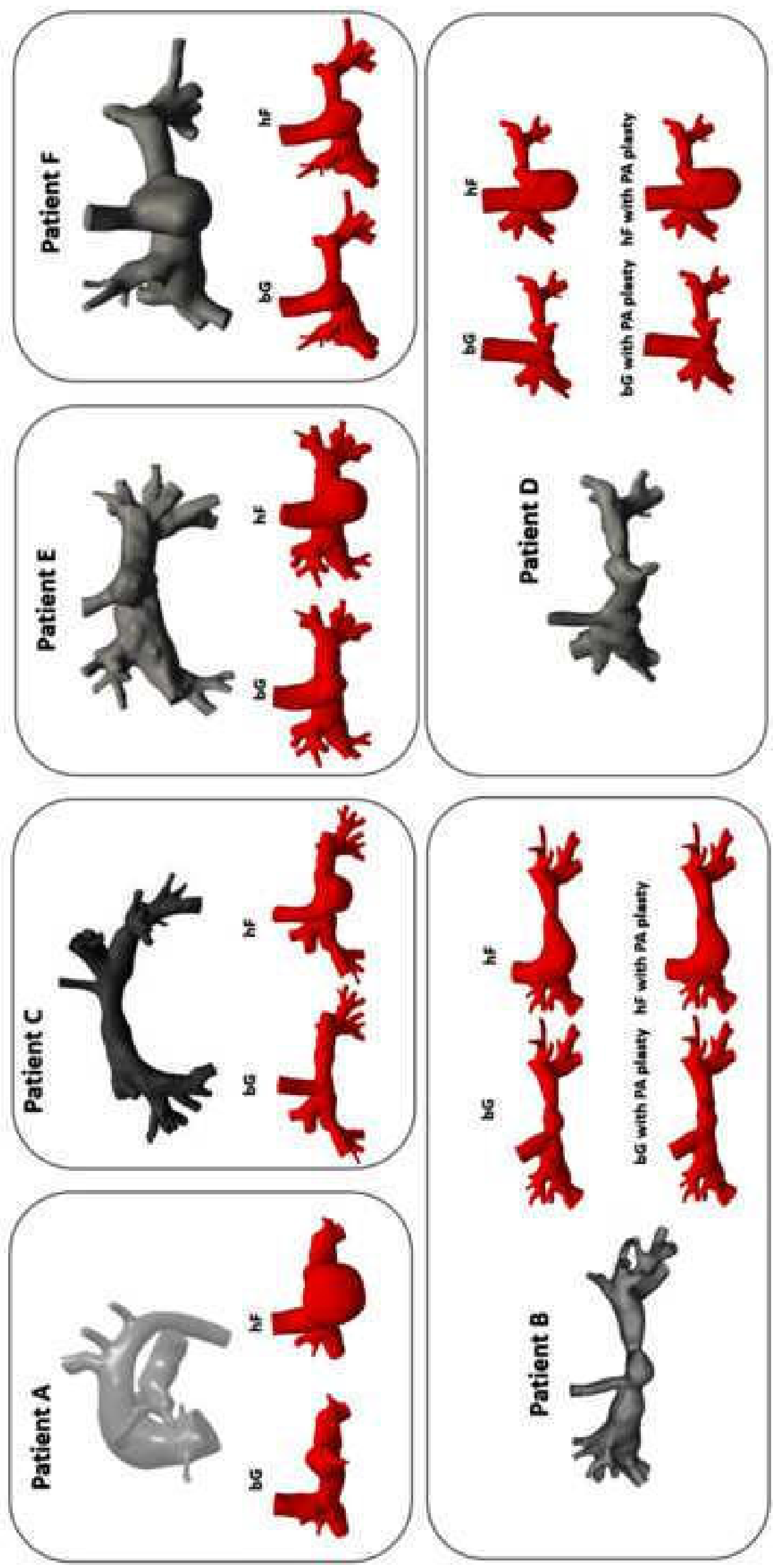

$\frac{\stackrel{0}{丂}}{i \frac{9}{4}}$ 


\section{Pressure}
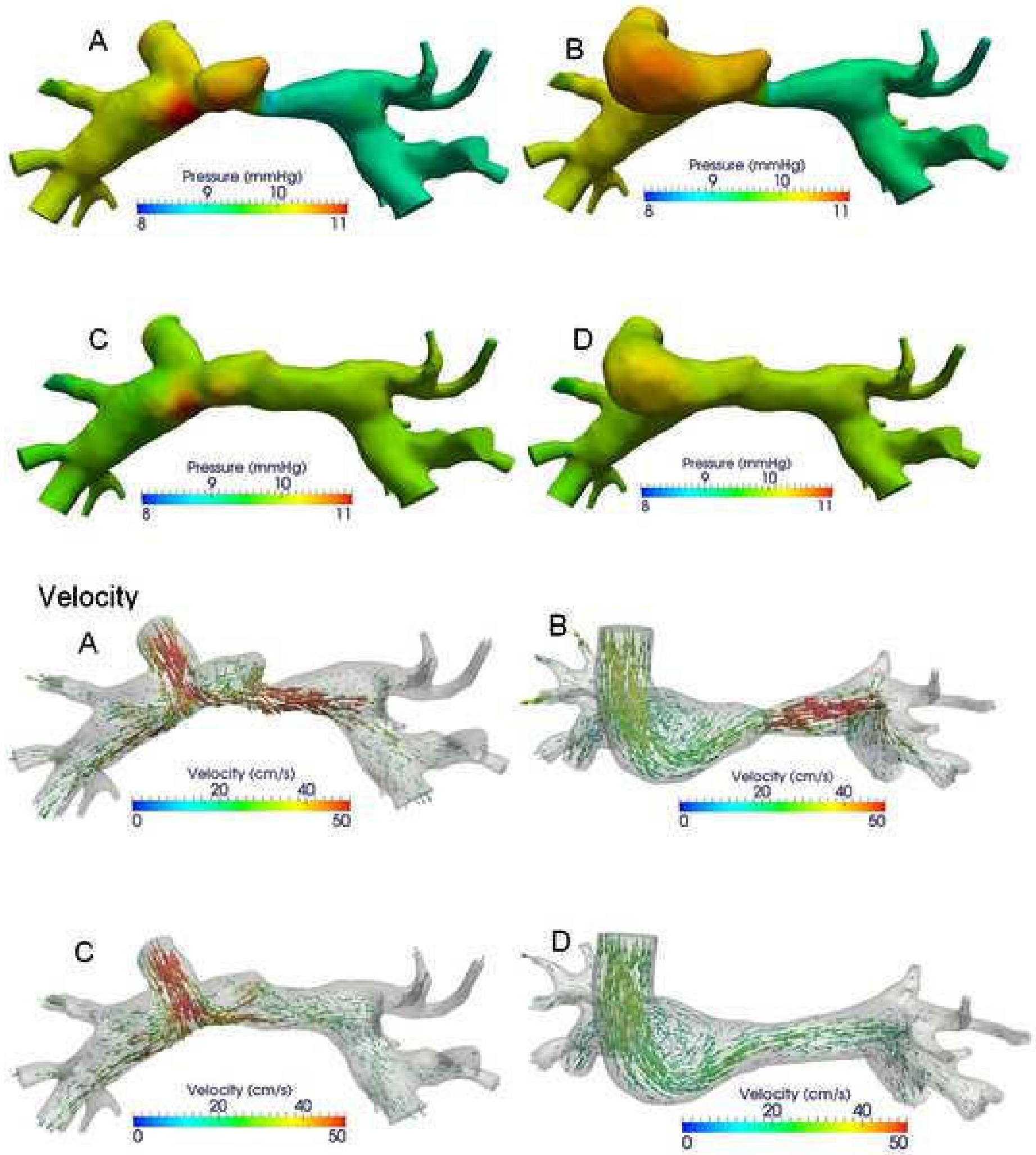


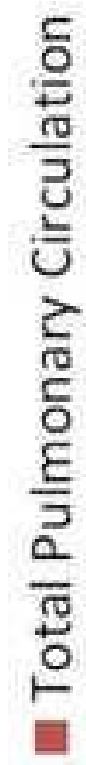

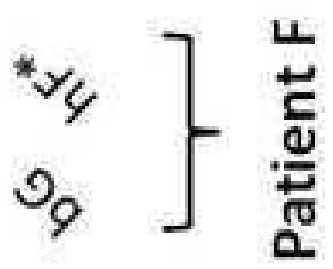

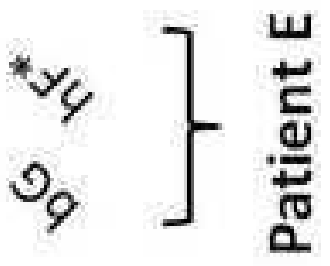

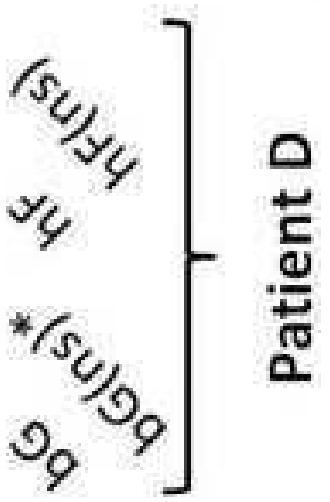

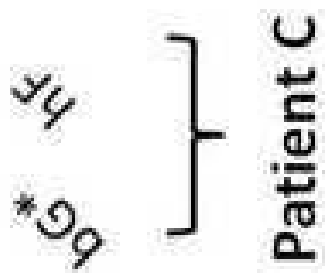

을

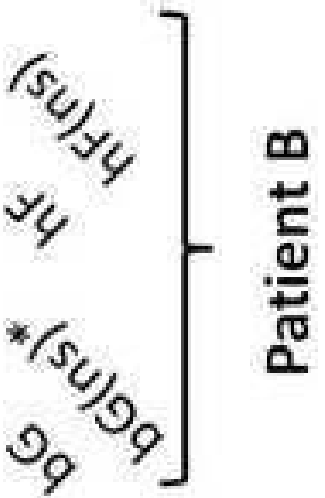

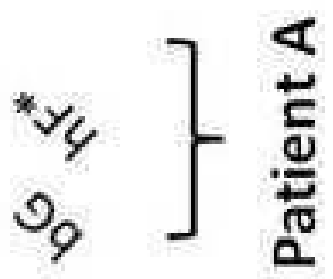

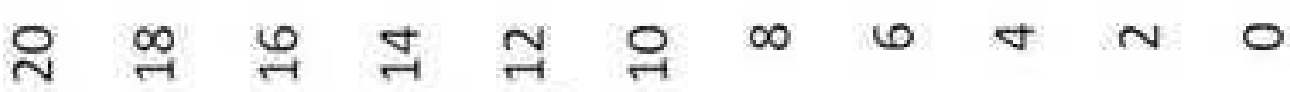

(MU) ssoך dəMOd

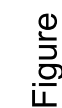

